\title{
ON THE FUELING OF MASSIVE BLACK HOLES AND THE PROPERTIES OF THEIR HOST SPHEROIDS
}

\author{
ANDRÉs EscAla \\ Departamento de Astronomía, Universidad de Chile, Casilla 36-D, Santiago, Chile. \\ Draft version May 13, 2018
}

\begin{abstract}
We study the relation between nuclear massive black holes and their host spheroid gravitational potential. Using simple models, we analyze how gas is expected to be transported in the nuclear regions of galaxies. When we couple it with the expected gas lifetime given by the Kennicutt-Schmidt Law, this naturally leads to the ' $\mathrm{M}_{\mathrm{BH}}-\mathrm{M}_{\mathrm{virial}}$ ' and ' $\mathrm{M}_{\mathrm{BH}}-\sigma$ ' relations.
\end{abstract}

Subject headings: quasars: general - galaxies: formation - black hole physics

\section{INTRODUCTION}

Most nearby massive spheroids (elliptical and spiral bulges) host nuclear massive black holes (MBH), whose mass scales as $\mathrm{M}_{\mathrm{BH}} \propto \sigma^{4-5}$ (the ' $\mathrm{M}_{\mathrm{BH}}-\sigma^{\prime}$ relation; Ferrarese \& Merritt 2000; Gebhardt et al. 2000). In recent years, several theories have been proposed to clarify the origin of this relation (Silk \& Rees 1998; Blandford 1999; Fabian 1999; Burkert \& Silk 2001; Zhao, Haehnelt \& Rees 2002; King 2003; Adams et al. 2003; Miralda-Escaudé \& Kollmeier 2005; Sazonov et al. 2005; Begelman \& Nath 2005). However, very little effort has been devoted to study how angular momentum is transfered in the centers of galaxies. This is an unavoidable challenge for the understanding of the formation of MBHs and the origin of the ' $\mathrm{M}_{\mathrm{BH}}-\sigma$ ' relation.

The study of $\mathrm{MBH}$ growth by gas accretion is usually focused on the study of accretion disks. However, these accretion disks are Keplerian by nature and therefore have neglible masses compared to that if the $\mathrm{MBH}$. They must be continously replenished, otherwise the mass of the MBH will not have a considerable growth. The key question in the growth of MBHs by accretion, is how to remove the large angular momentum of gas in a galaxy in order to funnel it into the accretion disk in the central sub-pc region: the so called 'Fueling Problem'. Without a proper understanding of this 'Fueling Problem', specially in the last few $100 \mathrm{pc}$, it is impossible to understand the growth of MBHs and to elucidate why their masses correlate with the host spheroid properties.

\section{MASS TRANSPORT IN GALACTIC NUCLEUS}

Several mechanisms for fueling gas from $\mathrm{R} \sim 10 \mathrm{kpc}$ down to $100 \mathrm{pc}$ have been proposed in the literature, including galaxy interactions, stellar bars and gravitational resonances (see Shlosman, Begelman \& Frank 1990 for a classic review, or Wada 2004 for a more recent one). However, gravitational torques in galaxy mergers arise as the dominant process for fueling large amounts of gas down to the central few hundred parsecs and for triggering most of the MBH growth. In a merger, after a violently relaxed core is formed at the center, most of the gas will settle in a nuclear disk (with typically several hundred parsecs in diameter) that is rotationally supported against the overall gravitational potential (Barnes 2002). The MBHs will migrate to the center, and merge in a timescale relatively short compared to the lifetime of the nuclear disk (Escala et al. 2004, 2005).

\subsection{Turbulent Disk}

Nuclear gas disks are characterized by being selfgravitating. It has been debated whether gravitational instability leads to disk fragmentation ('disk of clouds'; Shlosman et al. 1990) or to transient density fluctuations ('turbulent disk'; Wada 2004), depending on cooling/heating rates and thermal physics. I will mainly focus in the latter case because it is possible to advance further with analytical estimates.

A turbulence-dominated disk can be modeled on a zeroorder approximation as a steady $\alpha$-disk. We choose to parameterize the viscosity $\nu$ in terms of the turbulent speed $v_{\text {turb }}$ instead of the sound speed, because the turbulence in a nuclear disk is expected to be highly supersonic (Downes \& Solomon 1998) and therefore $v_{\text {turb }}$ becomes dynamically more relevant. In such a case, the disk height $h$ is $\mathrm{v}_{\text {turb }} / \Omega$ (being $\Omega$ the angular velocity) and the viscosity $\nu$, parameterized by Shakura and Sunyaev as $\alpha \Omega h^{2}$, becomes $\nu=\alpha v_{\text {turb }}^{2} / \Omega$. Therefore in a turbulence-dominated $\alpha$ disk with a flat rotation curve, the mass accretion rate can be written as

$$
\dot{\mathrm{M}}=\frac{2 \sqrt{2} \alpha \mathrm{v}_{\text {turb }}^{3}}{\mathrm{GQ}},
$$

where $\mathrm{Q} \equiv \mathrm{v}_{\text {turb }} \Omega / \pi \mathrm{G} \Sigma_{\mathrm{g}}$ is the 'turbulent' Toomre's parameter and $\Sigma_{g}$ is the gas surface density. Eq. (1) represents the mass accretion rate onto the inner accretion disk around the MBH (fueling).

In nuclear disks the turbulence can be driven by two mechanisms: feedback from star formation and selfgravity. The thickness of these nuclear disks is independent of whether or not the disk hosts a starburst (Jogee et al. 2005), suggesting that the turbulence is driven by selfgravity. We will now focus on the case where the turbulence is driven by gravitational instabilities and the energy is extracted from the disk rotation. Unfortunately, there is no consensus on the energy spectrum $\mathrm{E}(\mathrm{k}) \propto \mathrm{k}^{\mathrm{n}}$ for compressible supersonic turbulence (Ballesteros-Paredes et. al 2006). Boldyrev (2002) has presented a theoretical model suggesting that $\mathrm{n} \approx-1.74$ and that appears to be con- 
therein). Boldyrev's theory suggests that in the inertial range, compressible supersonic turbulence obey the Kolmogorov Law. In such a case the energy is transported in a cascade from the largest unstable scale characterized by the disk radius $R_{d}$ and rotation velocity $v_{r o t}$, to the turbulent eddies' scale characterized by the size $\lambda_{\text {turb }}$ and velocity $\mathrm{v}_{\text {turb. }}$. This can be expressed as

$$
\mathrm{v}_{\text {turb }} \sim \mathrm{v}_{\text {rot }}\left(\frac{\lambda_{\text {turb }}}{\mathrm{R}_{\mathrm{d}}}\right)^{1 / 3} .
$$

We shall also consider that the gas is in a disk in rotational support against the overall gravitational potential. For simplicity we assume that $\mathrm{v}_{\text {rot }}$ and $\sigma$ are constant with radius, that is only strictly valid for an isothermal sphere. On the central kiloparsec of galaxies, stellar bulges are the dominant components of the gravitational potential even in the most massive gaseous nuclear disks known so far, where observations (Downes \& Solomon 1998) found typically 5 times more mass in the bulge that in the gas $\left(\mathrm{M}_{\text {gas }} / \mathrm{M}_{\text {star }} \sim 1 / 5\right)$. Even in such an extreme case, the rotation velocity is $\mathrm{v}_{\text {rot }}=\left(1.2 \mathrm{G} \mathrm{M}_{\text {star }} / \mathrm{R}\right)^{1 / 2}=1.09 \sigma \approx \sigma$

Finally, combining $\mathrm{v}_{\text {rot }} \approx \sigma$ with Eqs. (1) and (2) we find that the mass accretion rate onto the inner Keplerian accretion disk is

$$
\dot{\mathrm{M}} \sim \frac{2 \sqrt{2} \alpha}{\mathrm{GQ}}\left(\frac{\lambda_{\text {turb }}}{\mathrm{R}_{\mathrm{d}}}\right) \sigma^{3} .
$$

Equation (3) shows that the mass transport in the nuclear regions of galaxies is controlled by the spheroid velocity dispersion $\sigma$ (the other factors are either dimensionless or physical constants). Therefore the rate at which the inner accretion disk around the MBH is replenished, strongly depends on the spheroid that hosts the $\mathrm{MBH}$.

\subsection{Disk of Clouds}

A disk composed by randomly moving clouds is the second possible model for the nuclear disk. The accretion flow in this 'disk', can be expressed in the same form of Eq. 1 replacing $\mathrm{v}_{\text {turb }}$ by the cloud velocity dispersion $\Delta \mathrm{v}$ (Goldreich \& Tremaine 1978). The formation of the clouds is driven by gravitational instabilities and their orbital energy is extracted from the disk rotation, as in the gravity-driven turbulence case. Therefore we expect that $\Delta \mathrm{v}$ scales with $\mathrm{v}_{\text {rot }}$ in a similar way as $\mathrm{v}_{\text {turb }}$ does, leading to an expression for the mass accretion rate analogous to Eq. 3. However, the exact value of $\Delta \mathrm{v}$ is highly uncertain and numerical simulations are required for an accurate estimation.

\section{CLOSURE EQUATION: GAS LIFETIME}

In order to determine the total mass accreted, we need to know for how long gas will be available to be accreted by the MBH.

Usually, AGN feedback is considered as the most likely physical process that determines the characteristic gas lifetime, expelling most of the gas in the host galaxy when the Eddington Limit is exceeded. However, in an inhomogeneous medium this limit can be greatly exceeded because photons tend to diffuse trough the low density medium. Several instabilities will produce inhomogeneities large enough to guarantee that the Eddington Limit is no longer valid, such as photon-bubble (Begelman 2001) or Kelvin-Hemboltz instabilities (Krumholz et. al 2005). Moreover on galactic scales, the ISM is endeed highly inhomogeneous and is hard to believe that most of the gas in a galaxy will be expelled due to AGN feedback.

On the other hand, these massive nuclear disks are characterized by an ongoing starburst and therefore gas depletion due to star formation will eventually be relevant in the absence of other processes. Although star formation is still poorly understood, it can be parameterized by a well constrained empirical relation called the KennicuttSchmidt Law (Kennicutt 1998) which can be stated as

$$
\dot{\Sigma}_{\text {gas }}=0.017 \frac{\Sigma_{\text {gas }}}{\tau_{\text {dyn }}}
$$

where $\dot{\Sigma}_{\text {gas }}$ is the star formation rate per unit area, $\Sigma_{\text {gas }}$ is the gas surface density and $\tau_{\mathrm{dyn}}=\mathrm{R}_{\mathrm{d}} / \mathrm{v}_{\text {rot }}$ is the dynamical (i.e. orbital) timescale of the gas. These nuclear disks have typical radius of $R_{d} \sim 0.1 R_{e}$, being $R_{e}$ the effective radius of the host spheroid. As shown in $\S 2$, the bulge dominates the overall gravitational potential $\left(\mathrm{v}_{\text {rot }} \approx \sigma\right)$ and therefore the dynamical timescale of the gas can be expressed as $\tau_{\text {dyn }}=0.1 \mathrm{R}_{\mathrm{e}} / \sigma$. Thus Eq. 4 implies that the gas lifetime $\mathrm{t}_{\text {gas }}$, defined as $\Sigma_{\text {gas }} / \dot{\Sigma}_{\text {gas }}$, is $\mathrm{t}_{\text {gas }}=\xi \mathrm{R}_{\mathrm{e}} / \sigma$ where $\xi \approx 5.9$.

The final mass of the $\mathrm{MBH}$ will be determined by $\mathrm{M}_{\mathrm{BH}}=\eta \dot{\mathrm{M}} \mathrm{t}_{\text {gas }}$, where $\eta$ is the fraction of gas in the inner accretion disk that finally ends inside the event horizon of the MBH. Using Eq. (3) the final MBH mass becomes

$$
\mathrm{M}_{\mathrm{BH}} \sim \frac{2 \sqrt{2} \eta \xi \alpha}{\mathrm{Q}}\left(\frac{\lambda_{\text {turb }}}{\mathrm{R}_{\mathrm{d}}}\right) \frac{\mathrm{R}_{\mathrm{e}} \sigma^{2}}{\mathrm{G}}
$$

which reproduces the correlation between $\mathrm{M}_{\mathrm{BH}}$ and the bulge mass $\mathrm{M}_{\text {virial }}=\mathrm{kR}_{\mathrm{e}} \sigma^{2} / \mathrm{G}$ (Marconi \& Hunt 2003; Haring \& Rix 2004), being $\mathrm{k}=8 / 3$ for an isothermal sphere.

Using the Faber-Jackson relation $\mathrm{R}_{\mathrm{e}} \propto \sigma_{\mathrm{e}}^{2}$ in Eq. 5, we obtain the standard ' $\mathrm{M}_{\mathrm{BH}}-\sigma$ ' form $\left(\mathrm{M}_{\mathrm{BH}} \propto \sigma^{4}\right)$. Since the ' $\mathrm{M}_{\mathrm{BH}}-\sigma$ ' and ' $\mathrm{M}_{\mathrm{BH}}$ - bulge virial mass' correlations have similar levels of scatter, the Faber-Jackson relation should not have larger scatter than either one in the galaxies where the correlations are measured.

Finally, we need to evaluate the zero point in Eq. 5. For gravity-driven turbulence $\alpha$ may be as large as 0.3 (Gammie 2001). In nuclear disks $\mathrm{v}_{\text {turb }} / \mathrm{v}_{\mathrm{rot}} \sim 1 / 5$ (Downes \& Solomon 1998), implying that $\lambda_{\text {turb }} / \mathrm{R}_{\mathrm{d}} \sim 0.008$ (Eq. 2 ). Using a typical value of $\mathrm{Q}=1.5$ (Martin \& Kennicutt 2001), we find $\mathrm{M}_{\mathrm{BH}} \approx 0.009 \eta \mathrm{M}_{\text {virial }}$. To reproduce the ' $\mathrm{M}_{\mathrm{BH}}$ - bulge virial mass' relation, we need to have $\eta \approx 1 / 6$. Thus, we need that less than $20 \%$ of the mass fed onto the inner accretion disk ends up inside the event horizon of the MBH.

\section{NUMERICAL STUDY}

The conclusions found in $\S 2$ and $\S 3$ are based in a steadystate model $(\alpha$-disk $)$. However, previous numerical studies on the mass transport in the inner $100 \mathrm{pc}$ of galaxies (Wada \& Norman 2002; Wada 2004) found that the mass accretion rate is highly time dependent with fluctuations of several order of magnitudes. For that reason, we have 
designed a simple numerical experiment to test the validity of the $\alpha$-disk model as a zero-order approximation.

In massive nuclear disks, self-gravity, cooling and feedback effects from star formation play a major role in determining the physics of the ISM and therefore must be included to accurately model the angular momentum and mass transport in the disk. However, a fully realistic model has multiple relevant parameters to be studied (such as the ones in the star formation prescription, heating/cooling processes, etc) and requires a long exploratory numerical study. This is beyond the scope of this Letter and will be studied in subsequent papers. For that reason, we decided instead to adopt an idealize model where star formation is not included and the thermodynamics is simplified by using an adiabatic Equation of State (E.O.S.), but where the velocity dispersion of the bulge $\sigma$ is the only varying parameter. With this E.O.S. we will not be able to compute accurate mass accretion rates, but we will be able to compare the changes in the accretion rates for different values of $\sigma$ and then reveal the role of $\sigma$ in the mass transport due to gravity-driven turbulence.

The model consist in a $\mathrm{M}_{\mathrm{g}}=5 \times 10^{7} \mathrm{M}_{\odot}$ gaseous disk around a $\mathrm{MBH}\left(\mathrm{M}_{\mathrm{BH}}=10^{7} \mathrm{M}_{\odot}\right)$ that is embedded in a stellar bulge. The MBH and the bulge are modeled as time-independent external potentials. For the bulge we use the potential of an isothermal sphere for $\mathrm{r} \geq 12 \mathrm{pc}$, and the potential of an homogenous sphere for $\mathrm{r}<12 \mathrm{pc}$. We modeled the MBH by a Plummer potential with core radius of $1 \mathrm{pc}$. We solved the hydrodynamic and Poisson equations using the FLASH adaptative mesh refinement hydrodynamics code (Fryxell et. al. 2000). The cartesian grid covers a $256^{2} \times 32 \mathrm{pc}^{3}$ region around the galactic center, with a spatial resolution of $2 \mathrm{pc}$. We use outflow boundary conditions.

The initial condition is a rotationally supported disk with uniform density profile, radius of $125 \mathrm{pc}$ and thickness of $4 \mathrm{pc}$. The initial temperature is set to $10^{3} \mathrm{~K}$ over the whole region. Random density and temperature fluctuations of less than $1 \%$ are added to the initial uniform disk. We performed four different runs where we vary the mass of the bulge, in such a way that the velocity dispersion of the bulge $\sigma$ is: $100,200,300$, and $400 \mathrm{~km} \mathrm{~s}^{-1}$. For a bulge modeled by an isothermal sphere, our rotationally supported disks have flat rotation curves. For example the run with the highest gas fraction $\mathrm{M}_{\text {gas }} / \mathrm{M}_{\text {star }}$ has a rotation velocity $\mathrm{v}_{\text {rot }}$ of $109 \mathrm{~km} \mathrm{~s}^{-1}$. This corresponds to the $\sigma=100 \mathrm{~km} \mathrm{~s}^{-1}$ run and the $\mathrm{M}_{\text {gas }} / \mathrm{M}_{\text {star }}$ ratio is similar to those found in the most massive gaseous nuclear disks (Downes \& Solomon 1998).

\subsection{Results}

Figure 1 illustrates a representative stage in the evolution of the system, showing the face-on density distribution at the plane of the disk for run 100 , at the time $t=$ 6 Myr. The figure shows a complicated multiphase and highly turbulent structure, in qualitative agreement with the findings of Wada \& Norman (2002).

As mentioned before, we start our simulation with an uniform disk with random fluctuations of less than $1 \%$ which are non-linearly amplified by self-gravity. The system is then let to evolve approximately for an orbital time $t_{\text {orb }}=2 R_{d} / v_{\text {rot }}$, in order to fully develop the turbulence in the disk. Because $t_{\text {orb }}$ varies among the different runs, we choose the longer $t_{\text {orb }}$ as starting point for our comparison. This corresponds to the run with $\sigma=100 \mathrm{~km} \mathrm{~s}^{-1}$ which has an orbital time $\mathrm{t}_{\text {orb }}=2.44 \mathrm{Myr}$.

Figure 2a shows the time evolution of the gas mass inside a radius of $2 \mathrm{pc}$ around the $\mathrm{MBH}$ (our resolution limit), for the four different values of $\sigma$ : 100 (black), 200 (red), 300 (blue) and $400 \mathrm{~km} \mathrm{~s}^{-1}$ (green). This figure shows a strong dependence on the bulge velocity dispersion, and the final gas mass $(\mathrm{r}<2 \mathrm{pc})$ varies by almost a factor 70 among the runs. In all the runs, the gas mass $(\mathrm{r}<2 \mathrm{pc})$ shows an approximately exponential growth.

In Fig. 2b, we plot the time evolution of the mass accretion rate inside $2 \mathrm{pc}, \dot{\mathrm{M}}(\mathrm{r}<2 \mathrm{pc})$, for the different runs and using the same color code as in Fig. 2a. The accretion rates are highly variable on timescales of $10^{5} \mathrm{yr}$ and with fluctuations of a couple of orders of magnitude in $\dot{\mathrm{M}}$, as found by Wada (2004). However, we found accretion rates considerably lower than those of Wada (2004). The origin of this discrepancy rely in the adiabatic E.O.S. This assumption produces unrealistically high Toomre's Q parameters compared to the observed ones $(\mathrm{Q} \sim 1.5$; Martin \& Kennicutt 2001), decreasing the mass accretion rates on Eq. 3.

Finally, in Fig. 3 we plot the average mass accretion rate $<\dot{\mathrm{M}}>$ as a function of $\sigma$. We compute $<\dot{\mathrm{M}}>$ simply as $\left[\mathrm{M}\left(\mathrm{t}_{\mathrm{f}}\right)-\mathrm{M}\left(\mathrm{t}_{\mathrm{i}}\right)\right] /\left[\mathrm{t}_{\mathrm{f}}-\mathrm{t}_{\mathrm{i}}\right]$, where $\mathrm{t}_{\mathrm{i}}$ and $\mathrm{t}_{\mathrm{f}}$ are the initial and final times. We choose $t_{i}=2.4 \mathrm{Myr}$ to ensure that all the runs have evolved for at least an orbital time $t_{\text {orb }}$ and at that time all the runs have almost the same gas mass inside $2 \mathrm{pc}$. The final time of the runs is $\mathrm{t}_{\mathrm{f}}=7 \mathrm{Myr}$. It is found that the average mass accretion rate $<\dot{M}>$ is strongly dependent on $\sigma$, as seen in Fig. 3. The black line is a least squares fit to the points in Fig. 3 and corresponds to $<\dot{\mathrm{M}}>\propto \sigma^{3.1}$. Therefore $<\dot{\mathrm{M}}>$ scales with $\sigma$ as expected from Eq. 3, despite the complex behavior of the gas (Fig. 1) and that the mass accretion rates are highly time-dependent (Fig. 2).

\section{DISCUSSION}

We perform a simple numerical experiment that illustrates the role of the spheroid gravitational potential on the mass transport in a gravity-driven turbulent disk. We find that -on average- the mass accretion rate behaves as predicted by the zero-order approximation $\left(\dot{\mathrm{M}} \propto \sigma^{3}\right)$, however our adiabatic simulations underestimate the mass accretion rates.

Using more realistic models by Wada \& Norman (2002) that found $\dot{\mathrm{M}} \sim 1.4 \mathrm{M}_{\odot} \mathrm{yr}^{-1}$ for an spheroid with $\sigma=100 \mathrm{kms}^{-1}$, we estimate that $\dot{\mathrm{M}} \approx$ $11.2\left(\sigma / 200 \mathrm{kms}^{-1}\right)^{3} \mathrm{M}_{\odot} \mathrm{yr}^{-1}$ (Eq. 3). If we assume that $10 \%$ of the mass fueled into the inner accretion disk is accreted by the $\mathrm{MBH}(\eta=0.1)$ and use the ' $\mathrm{M}_{\mathrm{BH}}$ $\sigma^{\prime}$ relation, we find that $\mathrm{MBHs}$ could gained a mass $\mathrm{M}_{\mathrm{BH}}$ in a rapid growth phase of $\mathrm{t}_{\text {growth }} \equiv \mathrm{M}_{\mathrm{BH}} / \eta \dot{\mathrm{M}} \approx$ $10^{8}\left(\mathrm{M}_{\mathrm{BH}} / 10^{8} \mathrm{M}_{\odot}\right)^{1 / 4} \mathrm{yr}$. This is in agreement with recent observations (Borys et al. 2005), that are consistent with a model where MBHs in submillimeter galaxies undergo a rapid growth to reach the local ' $\mathrm{M}_{\mathrm{BH}}-\sigma$ ' relation.

We find that the ' $\mathrm{M}_{\mathrm{BH}}-\sigma$ ' relation arises naturally from the fueling of either a turbulent disk or a 'disk of clouds' (coupled with the Kennicutt-Schmidt Law), and therefore 
is hard to distinguish between the two models. However, the zero point could be different allowing to discriminate between both disks. Also, the zero point allow us to address the possible role of star formation driven turbulence. Detailed numerical simulations of these various disks are necessary to determine a realistic zero point that could be compared with the one measured in the ' $\mathrm{M}_{\mathrm{BH}}-\sigma$ ' relation.
I thank Paolo Coppi for early stimulating discussions and access to Yale's HPC facilities. I thank Richard Larson, Diego Mardones and Paulina Lira for valuable comments. This research was funded by FONDAP grant 15010003. The software used in this work was in part developed by the DOE-supported ASC / Alliance Center for Astrophysical Thermonuclear Flashes at the University of Chicago.

\section{REFERENCES}

Adams, F. C., et al. 2003, ApJ, 591, 125

Ballesteros-Paredes, J., et al. 2006, astro-ph/0603357

Begelman, M. C. 2001, ApJ, 551, 897

Blandford, R. D. 1999, in Galaxy Dynamics, ASP 182, ed. Merritt

et. al. (San Francisco: Astron. Soc. Pacific), 87

Boldyrev, S. 2002, ApJ, 569, 841

Burkert, A., Silk, J. 2001, ApJ, 554, 151L

Downes, D., Solomon, P. M. 1998, ApJ, 507, 615

Escala, A., et al. 2004, ApJ, 607, 765

Escala, A., et al. 2005, ApJ, 630, 152

Fabian, A. C. 1999, MNRAS, 308, L39

Ferrarese, L., Merritt, D. 2000, ApJ, 539, 9

Fryxell, B., et al. 2000, ApJS, 131, 273

Gammie, C. F. 2001, ApJ, 553, 174

Goldreich, P., Tremaine, S. 1978, Icarus, 34, 227
Gebhardt, K., et al. 2000, ApJ, 539, 13

Haring, N., Rix, H. W. 2004, ApJ, 604L, 89

Jogee, S., Scoville, N. Z. \& Kenney, J. D. P. 2005, ApJ, 630, 837

Joung, M., Mac Low, M. 2006, astro-ph/0601005

Kennicutt, R. C. 1998, ApJ, 498, 541

King, A. 2003, ApJ, 596, 27L

Marconi, A., Hunt, L. K. 2003, ApJ, 589L, 21

Miralda-Escudé, J., Kollmeier, J. A. 2005, ApJ, 619, 30

Sazonov, S. Y., et al. 2005, MNRAS, 358, 168

Shlosman, I., Begelman, M., Frank, J. 1990, Nature, 345, 679

Silk, J., Rees, M. J. 1998, A\&A, 331, L1

Wada, K., Norman, C. A. 2002, ApJ, 566, L21

Wada, K. 2004, in Coevolution of Black Holes and Galaxies, ed L. C. Ho (Cambridge: Cambridge Univ. Press), 186

Zhao, H., Haehnelt, M. G., Rees, M. J. 2002, NewA, 7, 385 







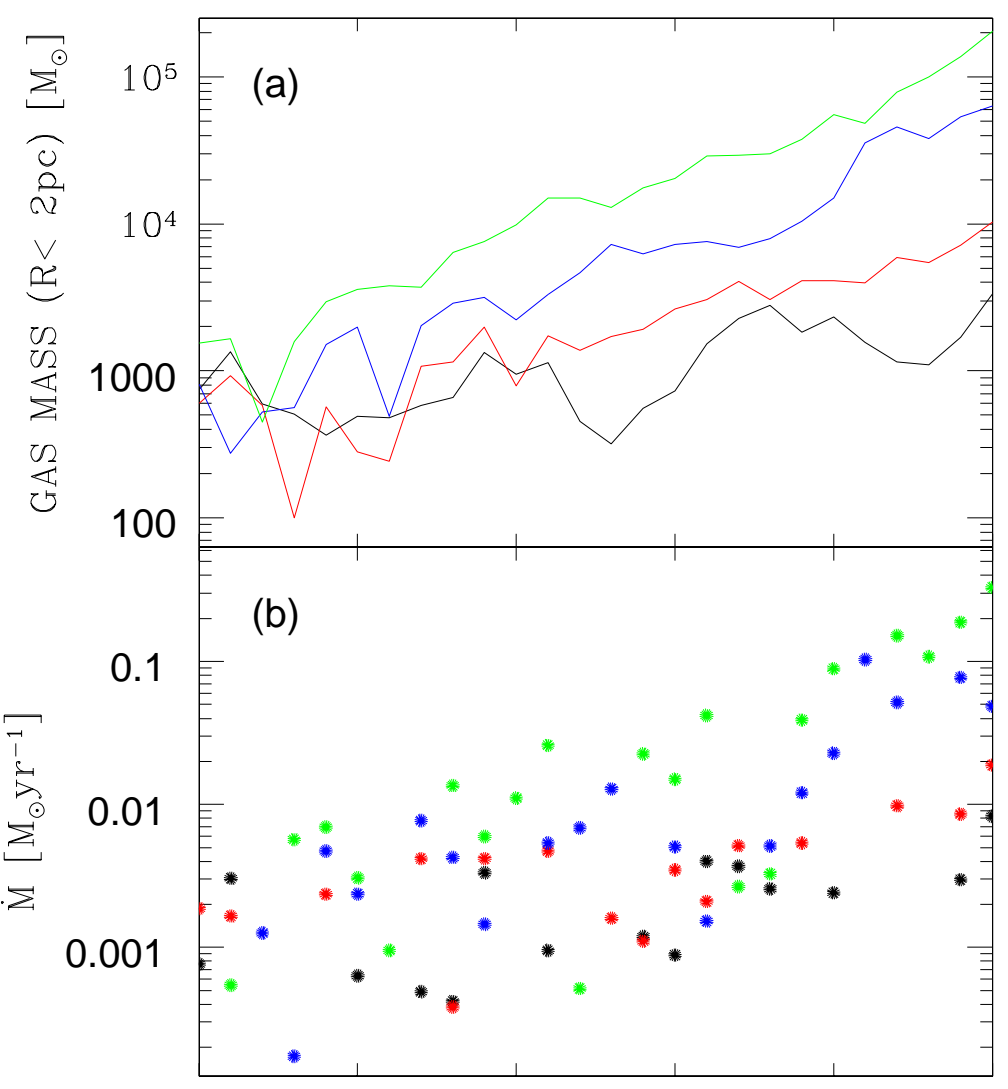

FIG. 2.- (a) Time evolution of the gas mass inside $2 \mathrm{pc}$ around the MBH (our resolution limit), for the four different values of $\sigma$ : 100 (black), 200 (red), 300 (blue) and $400 \mathrm{~km} \mathrm{~s}^{-1}$ (green). (b) Time evolution of the mass accretion rate inside $2 \mathrm{pc}, \dot{\mathrm{M}}(\mathrm{r}<2 \mathrm{pc}$ ), using the same color code as in (a). 


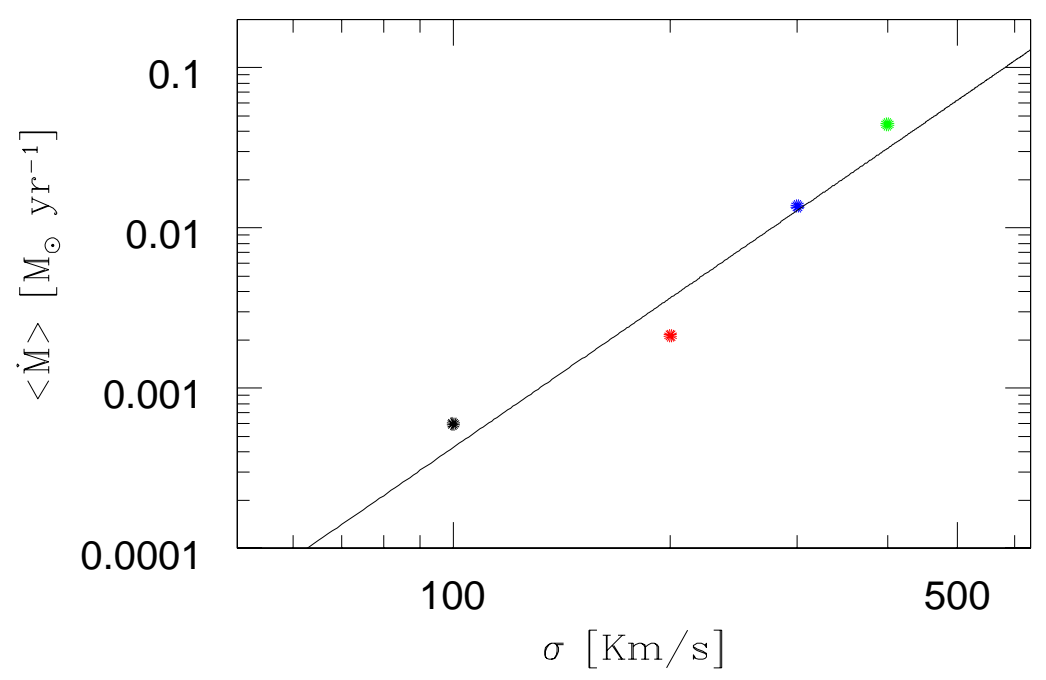

FIG. 3.- Mass accretion rate averaged over the time interval [2.4Myr,7Myr], for the four different values of $\sigma: 100,200,300$ and 400 $\mathrm{km} \mathrm{s}^{-1}$ (in the same color code as in Fig. 2). The black line is the best-fitting of the points using minimum least squares, and corresponds to $<\dot{\mathrm{M}}>\propto \sigma^{3.1}$. 